\title{
DIAGNOSTIC CHARACTERIZATION OF LASER-IRRADIATED DOT TARGETS
}

\author{
G. CHARATIS, G.E. BUSCH, B.K.F. YOUNG* and R.E. STEWART* \\ KMS Fusion, Inc., 3621 South State Road, Ann Arbor, MI 48106, \\ U.S.A. \\ - University of California, Lawrence Livermore National \\ Laboratory, Livermore, CA 94550, U.S.A.
}

\begin{abstract}
We have conducted a series of experiments at the KMS CHROMA facility using dot spectroscopy techniques to characterize uncertainties associated with spectral line ratio models commonly applied in determining electron temperatures and densities. Temperatures determined from the slope of the H-like free-bound continum and densities via holographic interferometry, are compared to line ratio methods. Dot targets of (typically $100 \mathrm{\mu mD} \mathrm{Mg}$ or Al) are irradiated with 2 to $40 \times 10^{13} \mathrm{~W} / \mathrm{cm}^{2}$ of $0.53 \mathrm{~mm}$ light. Time and spatial gradients are resolved using 4 diagnostics: a 4-frame holographic interferometer, an $x$-ray streak crystal spectrograph with a spatial imaging slit, a framing crystal $x$-ray spectrometer, and a conventional space-resolved time-integrating crystal spectrograph used for survey and calibration purposes. Preliminary results indicate the ionization distribution of these laser produced plasmas is not steady-state which plays an important role in measuring the temperature and and density. Electron temperatures derived from line-ratio techniques, assuming steady state conditions, disagree dramatically from simultaneous measurements using the slope of the H-like continuum. Electron densities using He-like triplet to singlet line ratios also differ from densities measured interferometrically.
\end{abstract}

\section{Experimental Scope}

Temperature and density measurements of hot plasmas determined from spectral line ratios can differ by large factors depending upon the assumptions used in the model. [1] Moreover, transient and non-uniform plasma distributions may contribute to ambiguous interpretations of available data. In this paper we present experimental measurements of $x$-rays emitted from laser-produced plasmas that are relatively free of spatial and temporal gradients. Using microdot target irradiation techniques 21 , in conjunction with time- and space-resolved diagnostics, we determine temperatures and densities by line ratio methods and by independent techniques. This comparison allows us to make clear tests of theoretical atomic models upon which the line ratio methods are based.

We have designed our experiments to measure $x$-ray emission from a small, localized volume of a laser plasma produced by irradiating a simple planar target. This was accomplished by using microdot targets composed of the element or elements to be studied on a low-z plastic substrate. The target was illuminated by a laser focal spot which was larger than the microdot. The surrounding carbon plasma emitted by the plastic substrate, collisionally confined the test ions emitted from the microdot into a slowly expanding plasma colum. For any cross-section of the plasma colum the plasma conditions are expected to be uniform. By viewing the plasma plume normal to the laser axis the axial gradient was resolved by using an imaging slit. The opacity was controlled by the density and diameter of the microdot test element. Temporal variations of the plume were detected using time-resolving diagnostic instruments. As a check on the well-known spectral line ratio methods for determining plasma conditions, electron densities were measured using holographic interferometry and temperatures were measured via the slope of $\mathrm{H}$-like free-bound continua.

Space- and Time-Resolved Diagnostics

Four principal plasma diagnostics were employed to study the plasmas. Spatial and temporal gradients were resolved using 3 diagnostics: a 4-frame holographic interferometer (4) provided non-spectroscopic measurements of the electron density, while temperatures (and densities) were measured with an x-ray streaked crystal spectrograph (XSCS) with a spatially imaging slit, and a 3 -frame crystal $x$-ray spectrometer (FCXS) $(5,6)$. These instruments were aligned to view the target edge-on, perpendicular to the laser axis. (See Fig.1) Additional plasma and laser diagnostics used included two space-resolved, time-integrating flat crystal mini-spectrometers; one using a high dispersion/high resolution crystal (ADP), while the other, with a lower dispersion crystal (KAP), was used as a survey instrument. Two x-ray pinhole cameras measured the focal spot size and the uniformity of the plasma. An optical 
streak camera recorded the CHROMA pulse shape for each shot. An array of photodiodes located inside the target chamber recorded the reflected laser light off the target, and with calibrated CrRoma optics the total absorbed energy for each shot was determined. The optical parameters were useful in hydrodynamic simulations of the laser produced plasmas.

\section{Preliminary Experiments}

We present preliminary results from ongoing data reductions as examples of the utility of our experiments. For all target shots, simultaneous imeasurements were made on all of the principal diagnostics. A variety of targets were irradiated including $\mathrm{Al}$ and $\mathrm{Mg}$ microdots. Typically, the microdots were $100 \mathrm{~m}$ diameter, mounted on a thick polystyrene substrate. The targets were irradiated with a single beam of 10 to $100 \mathrm{~J}$ of $530 \mathrm{~nm}$ light in a 1 ns long pulse (actually ten 100 ps pulses stacked in 100 ps intervals). The beam was focused on to the planar target surface to a diameter of 250 with a $F / 2.5$ lens resulting in irrarliances of $10^{13}$ to $10^{\mathrm{t}} \mathrm{W} / \mathrm{cm}^{2}$. The relative intensities of the measured spectra were corrected for film response, crystal reflectivities, filter transmissions, and photiscathode efficiencies. The corresponding holograms were reconstructed with a $C W$ Argon laser to produce interferograms which were Abel inverted to yield 4-frame density profiles at selected times during and after the CrRoma pulse duration.

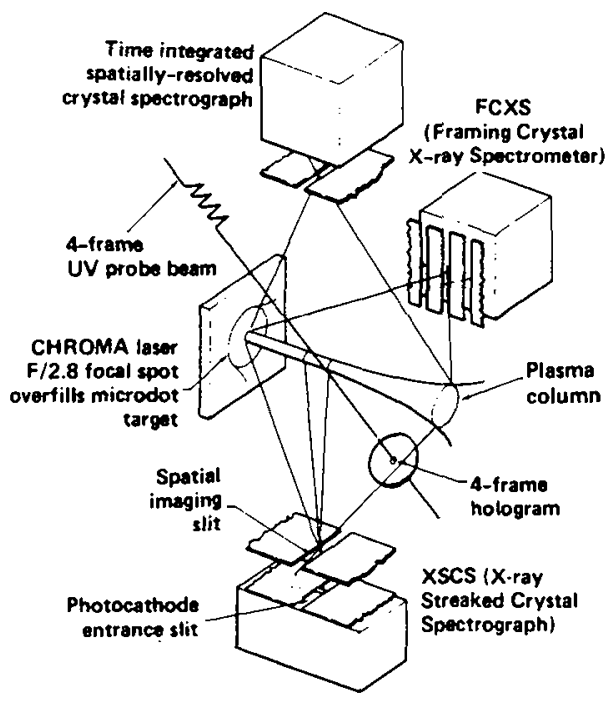

Figure 1. Schematic of the plasma diagnostics at the CrRoma laser target chamber.

\section{Electron Temperature Measurements}

Plasma electron temperatures, using both of the FCXS and XSCS instruments, in space and time, were mapped out from the slope of the H-like ion continuum [7] and from spectral line ratios. The line ratios included the He-like $1 s^{2}\left({ }^{\prime} \mathrm{s}\right)-1 \mathrm{~s} 3 \mathrm{p}\left({ }^{1} \mathrm{p}\right)$ to $\mathrm{H}-1$ ike $1 \mathrm{~s}-3 \mathrm{p}$ line ratio and also the H-like is-2p to He-like $1 \mathrm{~s} 2 \mathrm{p}\left({ }^{1} \mathrm{~g}\right)-2 \mathrm{p}^{2}\left({ }^{2} \mathrm{D}\right)$ dielectronic satellite line ratio. Using a steady state (CRE) atomic modeling code RATION/RATSHOW/SPECTRE, assuming an optically thin plasma, $[8]$ yielded dramatic differences in the temperature profiles, both in amplitude and shape, between the continuum slope and line ratio measurements. As seen in Fig.2, the line ratio determinations of electron temperature, whether optically thick or thin, differ significantly from the free-bound continuum measurement.

\section{Electron Density Measurements}

Electron density profiles measured spectroscopically were directly compared to those determined by holographic interferometry, both in space and in time. The densities determined spectroscopically were obtained via the He-like $1 \mathrm{~s}^{2}\left({ }^{2} s\right)-1 \mathrm{~s} 3 \mathrm{p}$ (' $\mathrm{p}$ )

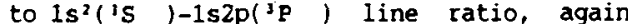
using the RATION/RATSHOW/SPECTRE model. The density profiles from an aluminum dot plasma are shown in Fig. 3. The magnesium target was irradiated with $10.0 \times 10^{13} \mathrm{~W} / \mathrm{cm}^{2}$. The density profiles at 1457 and $1657 \mathrm{ps,} \mathrm{with}$ respect to the beginning of the CHROMA pulse, indicate significant differences between the interferometrically and optically thin (or thick) spectroscopicaliy determined density profiles. When, arbitrarily, we include optical thickness and assume the spot size to be $50 \mu \mathrm{mD}$, and $60 \%$ of the plasma electrons are due to background, then the density profile approaches the interferometric measurement.

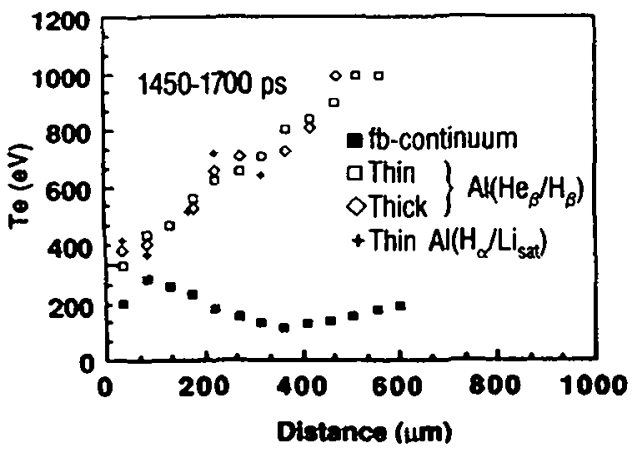

Figure 2. Comparison of temperature profiles determined from the free-bound continuum and by using steady-state line-intensity ratio techniques. (FCXS data at 1450-1700ps) 
Electron Density - Al dot CH 1.1e14 W/cm2

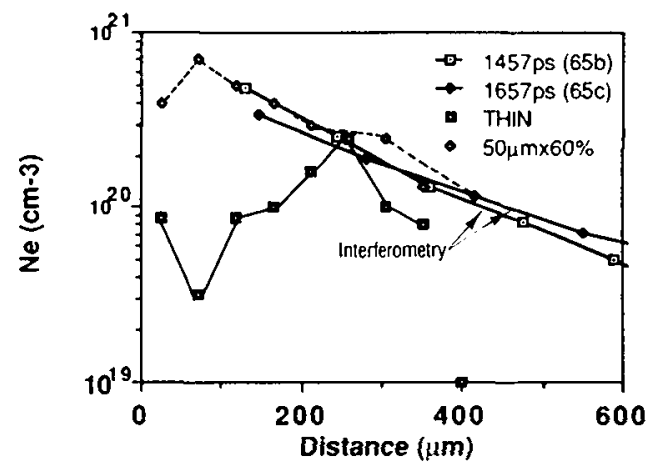

Figure 3. Comparison of electron density profiles determined by interferometry and by the ratio of the He-like $1 s^{2}\left({ }^{2} S\right)-1 s 3 p\left({ }^{2} \mathrm{P}\right)$ to $1 s^{2}\left({ }^{2} S\right)-1 s 2 p\left({ }^{3} P\right)$ lines. (FCXS data)

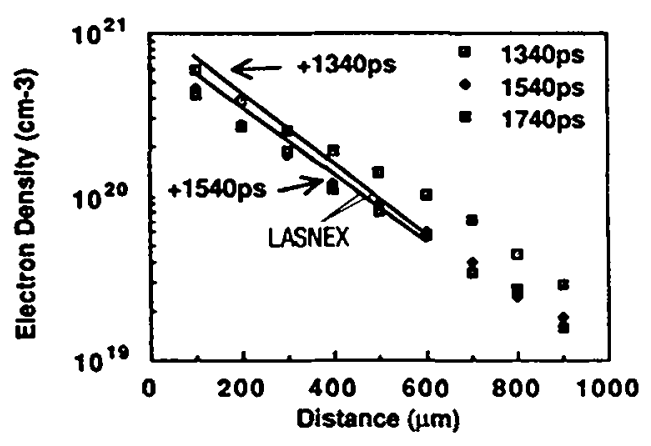

Figure 4. Electron density profiles are predicted reasonably well by a 2-D LASNEX hydrodynamics simulation.

Hydrodynamic Modeling Code Comparisons

Electron density and temperature profile measurements in both space and time ideally allow for the direct comparison to available hydrodynamic modeling codes, if the atomic physics data base in them is correct. In that case, the code predictions depend principally on the hydrodynamic aspects of the simulations. Interferometrically measured density profiles are modeled fairly well by LASNEx code simulations[9], as shown in Fig.4. It is also possible, to apply the XRASER/SPECTRE code to post-process the LASNEX density and temperature predictions yielding synthetic spectra from which line ratio comparisons can be made with experiments. As an example, we compared the predicted ratios of several resonance lines computed using LASNEX/XRASER/SPECTRE, with experiments for a Mg plasma irradiated with $7 \times$ $10^{13} \mathrm{~W} / \mathrm{cm}^{2}$ (see Figs.5 and 6). Agreement between the simulated and experimental line ratios is mixed 191. LASNEX/XRASER line ratio simulations for ratios usually applied in temperature measurements agree well with XSCS measurements, as seen in Fig.5. On the other hand, Fig.6 shows that line ratio LASNEX/XRASER simulations of lines used in density measurements do not agree well with FCXS data.
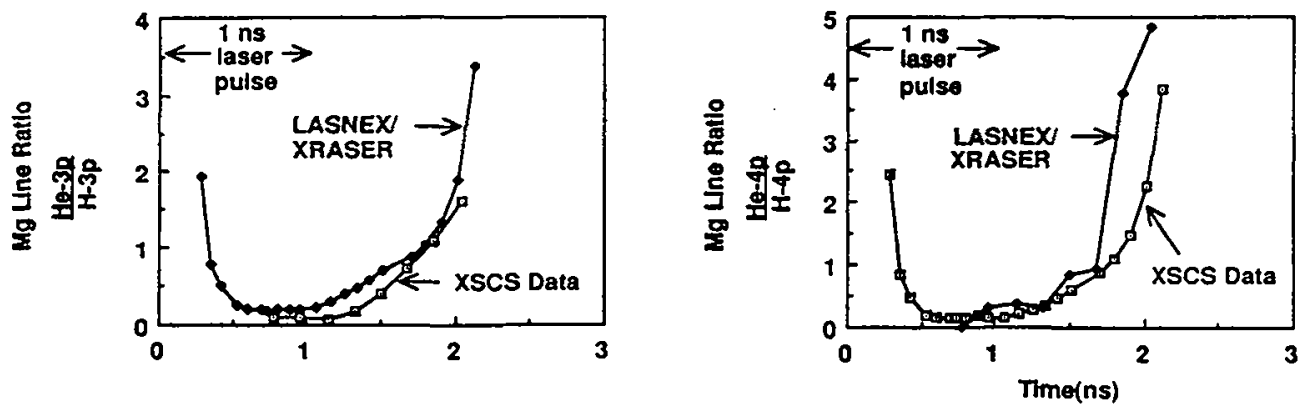

Figure 5. Comparison of XSCS data and LASNEX/XRASER/SPECTRE simulations show good agreement for $M g$ resonance line ratios He $\left(1 s^{2}-1 s 3 p\right) / H(1 s-3 p)$ and $\mathrm{He}\left(1 \mathrm{~s}^{2}-1 \mathrm{~s} 4 \mathrm{p}\right) / \mathrm{H}(1 \mathrm{~s}-4 \mathrm{p})$. XsCS viewed a 200-350 $\mathrm{mm}$ region from a Mg dot plasma heate by $7 \times 10^{3} \mathrm{~W} / \mathrm{cm}^{2}$. 
(a)

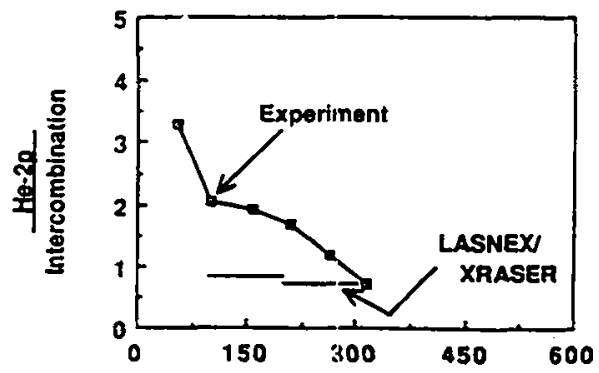

(b)

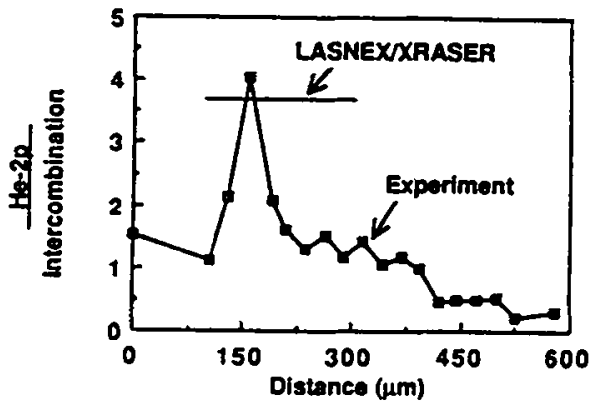

Figure 6, Comparison of the He-like resonance to intercombination line ratios measured with the FCXS to LASNEX/XRASER/SPECTRE calculations show poor agreement. (a) at $1350-1600$ ps and (b) at $1850-2100$ ps.

Summary

Time- and space-resolved $x$-ray spectra have been measured simultaneously for the first time from laser-i rradiated miçrodot targets. Electron temperatures and densities determined by 1 ine ratio techniques are shown to differ significantly from independent non-line ratio measurements. Optical thickness and the application of steady-state atomic physics simulations may play a role in the obvious differences observed. Non-stationary atomic models, currently in development, will be applied to the present data.

This work was performed in part under the auspices of the U.S. Dept. of Energy by Lawrence Livermore National Laboratory under Contract No.W-7405-ENG-48 and in part under the U.S. Dept. of Energy by KMS Fusion, Inc. under contract DE-AC08-82-DP40152.

\section{References}

1. S.R.Stone and J.C.Weisheit, UCID-20262 LLNL (1984)

2. M.J.Herbst, et al., Rev. Sci. Instrum. 53(9), 1418-1422 (1982)

3. P.G.Burkhalter et al., Phys. Fluids 26(12), 3850-3859 (1983)

4. Gar.E.Busch, et al., Rev. Sci. Instrum. 56(5), 879-884 (1985)

5. B.K.F.Young, et al., Rev. Sci. Instrum. 57(11), 2729-2732 (1986)

6. D.H.Auston, Appl. Phys. Lett. 26, 101 (1975)

7. C.DeMichelis and M.Mattioli, Nuc1. Fusion 21(6), 677-754 (1981)

8. R.W.Lee, J.Quant. Spectrosc. Radiat. Transf. 31, 91-101 (1984)

9. M.D. Rosen, private commication. 